\title{
CYCLICAL MICROMOVEMENT AND FRACTURE HEALING
}

\author{
M. H. H. NOORDEEN, C. B. D. LAVY, N. S. SHERGILL, J. D. TUITE, A. M. JACKSON \\ From the Royal National Orthopaedic Hospital, Stanmore, England
}

We studied 56 patients with fractures of the tibial shaft in a multicentre prospective randomised trial of three methods of external fixation. Group I was treated with a fixator which was unlocked at 4 to 6 weeks to allow free axial compression (axial dynamisation) with weight-bearing. Group II was treated with a fixator that was similarly unlocked at 4 to 6 weeks but included a small silicone spring which on weight-bearing could be compressed by up to $2 \mathrm{~mm}$. This spring returns to its original length on cessation of weight-bearing thus allowing cycles of motion of up to $2 \mathrm{~mm}$. Group III had a spring fixator like group I, but it was unlocked from the start to allow cyclical micromovement as soon as weight-bearing began. Fracture healing was monitored by the measurement of fracture stifiness.

We defined healing as achieving a stifiness of $15 \mathrm{Nm}$ per degree. The mean time was 14.1 weeks in group I, 15.9 weeks in group II, and 19.3 weeks in group III. The difference between groups was statistically significant ( $p=0.004)$. The $95 \%$ confidence intervals for the average delay in healing with early cyclical micromovement (group III) as compared with later axial dynamisation (group I) was 1.8 to 8.7 weeks. The healing time in patients whose cyclical micromovement was delayed for 4 to 6 weeks (group II) was between these two extremes, but the differences from either of the other groups could have been due to patient selection.

In the patients who completed the full trial, there were pin-track infections in over $60 \%$ of those in the cyclical micromovement groups compared with $20 \%$ in the axial dynamisation group $(p=0.03)$. In addition to this difference, nine patients required early removal of

M. H. H. Noordeen, MCh Orth, FRCS, Consultant Orthopaedic Surgeon N. S. Shergill, FRCS. Zimmer Fellow

The Royal National Orthopaedic Hospital Trust, Brockley Hill, Stanmore, Middlesex HA7 4LP, UK.

C. B. D. Lavy, MCh Orth, FRCS, Consultant Orthopaedic Surgeon The Middlesex Hospital, Mortimer Street, London WIN 8AA, UK.

J. D. Tuite, FRCS, Consultant Orthopaedic Surgeon

Broomfield Hospital, Court Road, Broomfield, near Chelmsford, Essex CM15 8DR, UK.

A. M. Jackson, FRCS, Consultant Orthopaedic Surgeon

St Mary's Hospital, Roehampton, London, UK.

Correspondence should be sent to Mr M. H. H. Noordeen.

(C)1995 British Editorial Society of Bone and Joint Surgery 0301-620X/95/41015 \$2.00 their fixator because of pin-track infection or loosening: all but one of them was in a cyclical micromovement group.

Our study suggests that cyclical micromovement during the weight-bearing period of fracture healing delays fracture union.

J Bone Joint Surg [Br] 1995:77-B:645-8.

Received 27 April 1994; Accepted after revision II November 1994

Many authors have suggested that some movement at a fracture site encourages healing (McKibbin 1978; De Bastiani, Aldegheri and Brivio 1984; Goodship et al 1987). Kenwright et al (1991) showed that early cyclical compression and distraction micromovements of up to $1 \mathrm{~mm}$ increase the rate of fracture healing in tibial osteotomies in sheep and, clinically, in fractures of the tibia.

We have investigated the influence of cyclical micromovement continued throughout the whole course of fracture treatment.

\section{PATIENTS AND METHODS}

From February 1990 to July 1993 all patients with fractures of the tibial shaft at the four participating hospitals were prospectively considered for the trial. Patients were excluded if they were under 16 or over 65 years of age, the fracture was less than $5 \mathrm{~mm}$ from a joint, it was through pathological bone, or was of a simple transverse type (AOA3). Since these fractures are often undisplaced, they are easily treated in a cast, and would need distraction to allow axial movement. Patients were also excluded if they were unwilling to give informed consent or were unable to comply with the protocol which required weight-bearing on their fixator.

A total of 56 patients, 20 women and 36 men, entered the trial. Their fractures were classified according to the AO system and graded according to Lange's modification of Gustilo's system (Lange et al 1985). Randomisation was into three groups using a sealed-envelope system stratified to ensure that similar numbers of fractures of each severity were entered into each group.

All patients had an Orthofix external fixator (Orthofix SRL, Verona, Italy) applied within 96 hours of the injury using a standard method with two pins above and two pins below the fracture. The three types of external fixator and the management of each group were as follows:

Group I. These patients received a standard Orthofix 
external fixator with a locking nut which allowed later axial dynamisation. This was locked for four weeks in all cases and released later when radiographs showed signs of callus formation, at either four or more weeks.

Group II. These patients had a modified fixator which contained a silicone cushion. This allowed the fixator to shorten by $2 \mathrm{~mm}$ under a deforming force of $200 \mathrm{~N}$ and return to its original length when the force was released. The fixator was kept locked for four or more weeks as for group I until a radiograph showed callus.

Group III. These patients had a cushioned fixator like that in group II, but it was left unlocked from the time of its application.

All patients were encouraged to bear weight as soon as possible, at least within three days, and were discharged from hospital when they had achieved this and were comfortable.

We assessed the patients in a special follow-up clinic at weeks $1,2,4,6,8$ and 12 , and then every two weeks until union. At each visit pin sites were inspected, and radiographs were taken every four weeks. The fixators were unlocked according to the protocols outlined above. If no callus had appeared by 12 weeks then the surgeon had the option of removing his patient from the trial and performing a bone graft.

Fracture stiffness was measured every two weeks by removing the fixator bar and then using a force plate and a strain gauge. Union was defined as a stiffness of $15 \mathrm{Nm} /$ degree (Richardson et al 1994). When this had been achieved the fixator was removed but the patient remained under follow-up for at least three months.

Statistical methods. Randomisation to treatment was by a set of random permuted blocks, arranged to improve the chances of balance across hospitals and the groups of fracture severity. The analysis of healing times was confined to those patients who completed the trial according to protocol. The possible effect of the exclusions is considered in the results section.

Computation used the program Minitab version 8.1, and the difference between treatment groups for healing time was assessed by the analysis of variance. The confidence intervals which we quote include the Tukey correction for multiple comparisons, using the procedure 'oneway'. Differences in healing times between groups were also analysed after making allowance for age as a linear covariate, and the AO group, the hospital and the Lange grouping as factors, using the procedure 'glm'. The significance levels for differences in proportion of pin-track infections were computed using chi-squared tests or Fisher's exact test.

\section{RESULTS}

Forty-one of the 56 patients completed the trial. Nine developed pin-track sepsis which required the early removal of pins, three withdrew their consent during the trial, one emigrated, one had an unrecognised fracture extending into a joint, and one patient was uncontactable (Table I). Another five patients, three in group I and two in group II, completed the trial but had omissions in their protocol and were excluded from analysis. We were finally able to analyse the results in 36 patients (Table II) and Figure 1.

The difference between treatment groups for time to union was statistically significant $\left(p=0.004, F_{2.33}=6.58\right)$. The 95\% confidence interval for the difference between group I and group II was 1.7 to 8.8 weeks, but the confidence intervals for differences between groups I and II and groups II and III overlapped. After allowing for imbalances in the severity of the fracture and the age of the patients, further analysis increased the significance level between group I and group III to $p=0.0001$ and increased the difference between groups I and II and between groups II and III by a further 1.1 weeks.

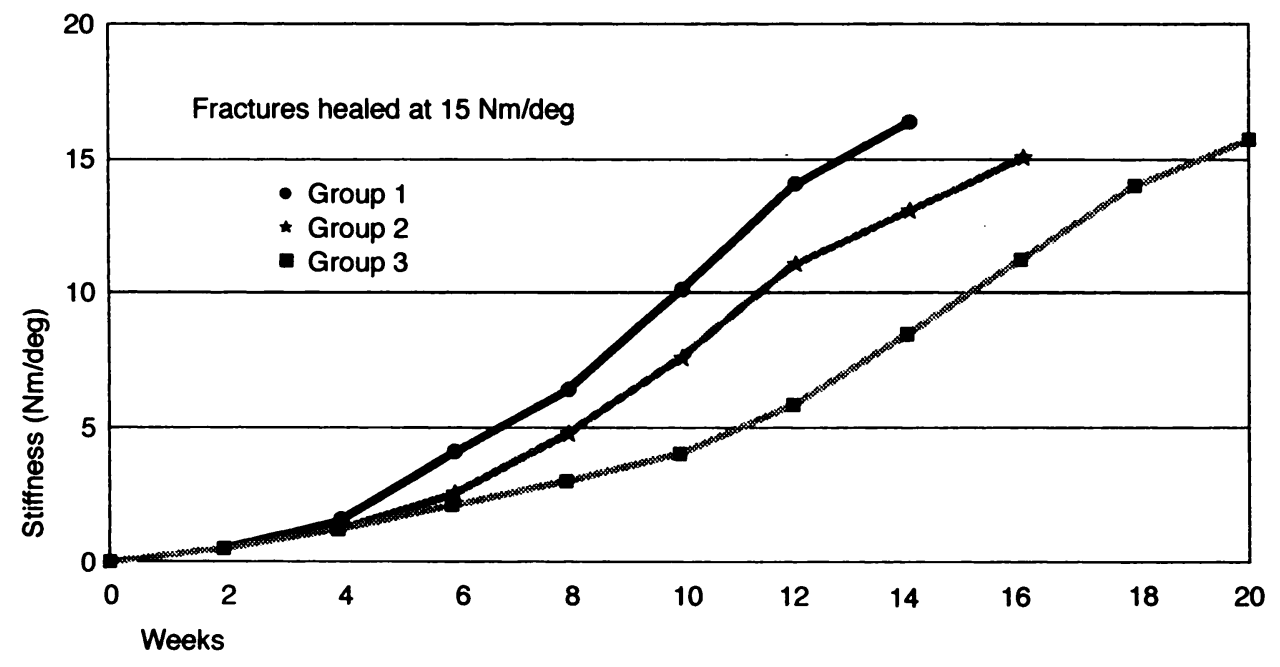

Fig. 1

Mean stiffness for each of three groups at two-weekly intervals. 
Table I. Details of the 15 patients excluded

\begin{tabular}{llllll}
\hline Groups & Sepsis & Emigrated & Withdrew & Uncontactable & $\begin{array}{l}\text { Fracture } \\
\text { joint }\end{array}$ \\
\hline I & 1 & 1 & 0 & 0 & 0 \\
II & 5 & 0 & 2 & 1 & 0 \\
III & 3 & 0 & 1 & 0 & 1 \\
\hline
\end{tabular}

Table II. Details of patients included in the trial

\begin{tabular}{|c|c|c|c|}
\hline & Group I & Group II & Group III \\
\hline Original number & 19 & 18 & 19 \\
\hline Fixator removed early & 1 & 5 & 3 \\
\hline $\begin{array}{l}\text { Excluded for other } \\
\text { reasons (see text) }\end{array}$ & 4 & 5 & 2 \\
\hline Final number analysed & $14(12 \mathrm{~m}: 2 \mathrm{f})$ & $8(7 \mathrm{~m}: 1 \mathrm{f})$ & $14(12 \mathrm{~m}: 2 \mathrm{f})$ \\
\hline $\begin{array}{l}\text { Mean age in years } \\
\text { (range) }\end{array}$ & $30.9(17$ to 65$)$ & $28.5(19$ to 36$)$ & $31.5(17$ to 54$)$ \\
\hline $\begin{array}{l}\text { Mean time to union } \\
\text { in weeks (standard } \\
\text { error) }\end{array}$ & $14.1(1.02)$ & $15.9(1.4)$ & $19.3(1.02)$ \\
\hline $\begin{array}{l}\text { Pin track sepsis \% } \\
\text { (approximate } \\
\text { standard error) }\end{array}$ & $21(11)$ & $62(17)$ & $64(13)$ \\
\hline
\end{tabular}

The incidence of pin-track sepsis was similar in both the cyclical micromovement groups II and III; when these were combined the incidence was significantly greater than in the axial dynamisation group I $(p=0.03)$. In addition to these episodes of pin-track sepsis nine patients were excluded from the trial because of pin loosening or pin-track infection which resulted in early removal of the external fixator. Only one of these nine patients was in the axial dynamisation group I, five were in group II and three in group III. This indicates an increased risk associated with this type of cyclical micromovement.

A fracture stiffness of $15 \mathrm{Nm} /$ degree proved to be a useful measure of union. Of the 41 patients who completed the trial on this basis, only one suffered refracture in a fall three weeks after removal of the fixator. The fracture was undisplaced and was treated satisfactorily in a plaster for three weeks.

\section{DISCUSSION}

Fracture healing is a complex process which may be influenced by many factors. There is evidence that loading a fracture to encourage interfragmentary motion stimulates callus formation, consolidation and osteogenesis (Rubin and Lanyon 1984). Despite many papers on the properties of fixation devices and the relationship of fracture motion to the nature of the healing process, the optimal magnitude, direction and timing of such movement have still to be clarified.

Our study has shown that allowing up to $2 \mathrm{~mm}$ of axial cyclical micromovement on weight-bearing produced a mean delay in healing of three weeks compared with a fixator that was locked for four to six weeks, then subsequently allowed $2 \mathrm{~mm}$ of micromovement, and of about six weeks compared with a fixator locked for four to six weeks and allowing axial compression only. It seems clear that a maximum of $2 \mathrm{~mm}$ of cyclical micromovement during weight- bearing retarded fracture healing.

Aro and Chao (1993) have shown in canine tibiae that close apposition of fracture fragments by axial dynamisation decreases the fracture gap and facilitates healing. This is supported by our work which shows that the axial dynamisation in group I, tending to close the fracture gap, allowed faster healing than the micromovement in groups II and III. The presence of a gap in a fracture under treatment in a locked fixator also encourages some micromovement due to pin bending and movement at the pin-bone interface. Any such fracture gap would tend to close on weightbearing but reopen after cessation of weight-bearing by elastic recoil. Simple axial dynamisation would tend to minimise this gap. Aro and Chao (1993) also showed that axial dynamisation decreased the load-sharing of the external fixator and increased load transmission through intact bone. This reduced pin-bone interface stresses and the rate of pin loosening and pin-track infections in dog tibiae. This difference was confirmed in our study, which showed a significantly lower rate of pin-track complications in the patients treated by axial dynamisation.

Wallace et al (1993) have shown that in the early phase of fracture healing, a periosteal collateral circulation pre- 
dominates; obliteration of this network has a profound effect on healing. The deleterious effect of $2 \mathrm{~mm}$ of micromovement may be due to the disruption of this delicate developing vascular network.

Kershaw, Cunningham and Kenwright (1993) have shown that the application of micromovement at an early stage in the fracture healing may reduce the time to union. At first this seems to contradict our results, but they used micromovement of the order of $0.7 \mathrm{~mm}$ only, and it was provided by a pneumatic activator during the first few days

\section{REFERENCES}

Aro HT, Chao EYS. Bone healing patterns affected by loading, fracture fragment stability, fracture type and fracture site compression. Clin Orthop 1993;293:8-17.

De Bastiani G, Aldegheri R, Brivio LR. The treatment of fractures with a dynamic axial fixator. $J$ Bone Joint Surg [Br] 1984;66-B:538-45.

Goodship AE, Kelly DJ, Rigby HS, Watkins PE, Kenwright J. The effect of different regimes of axial micromovement on the healing of experimental tibial fractures. Orthop Trans 1987;11:285.

Kenwright J, Richardson JB, Cunningham JL, et al. Axial movement and tibial fractures: a controlled randomised trial of treatment. J Bone Joint Surg [Br] 1991;73-B:654-9.

Kershaw CJ, Cunningham JL, Kenwright J. Tibial external fixation, weight bearing and fracture movement. Clin Orthop 1993;293: 28-36. after fracture and before weight-bearing had started. The micromovement allowed in our trial was greater and occurred only during weight-bearing. It seems that micromovement very early in the natural history of a fracture encourages healing, but that late micromovement, perhaps after the establishment of a vascular network, is deleterious.

No benefits in any form have been received or will be received from a commercial party related directly or indirectly to the subject of this article.
Lange RH, Bach AW, Hansen ST Jr, Johansen KH. Open tibial fractures with associated vascular injuries: prognosis for limb salvage. J Trauma 1985;25:203-8.

McKibbin B. The biology of fracture healing in long bones. J Bone Joint Surg [Br] 1978;60-B:150-62.

Richardson JB, Cunningham JL, Goodship AE, O'Connor BT, Kenwright $\mathbf{J}$. Measuring stiffness can define healing of tibial fractures. $J$ Bone Joint Surg [Br] 1994;76-B:389-94.

Rubin CT, Lanyon LE. Regulation of bone formation by applied dynamic loads. J Bone Joint Surg [Am] 1984;66-A:397-402.

Wallace AL, Draper ERC, Strachan RK, McCarthy ID, Hughes SPF. The vascular response to micromovement in experimental fractures. In: Turner-Smith AR, ed. Micromovement in orthopaedics. Oxford: Clarendon Press, 1993. 